\title{
Pancreatitis del surco (groove) que imita cáncer de páncreas: presentación de un caso y revisión de la literatura
}

\section{Groove pancreatitis mimicking pancreatic cancer: Case report and literature review}

\author{
Julián Ernesto Parga-Bermúdez, ${ }^{*}$ (1) Martín Alonso Gómez-Zuleta. ${ }^{2}$ []
}

\author{
Gacceso abierto \\ Citación: \\ Parga-Bermúdez JE, Gómez-Zuleta MA. \\ Pancreatitis del surco (groove) que imita \\ cáncer de páncreas: presentación de un \\ caso y revisión de la literatura. Rev Colomb \\ Gastroenterol. 2021;36(Supl.1):19-25. https://doi. \\ org/10.22516/25007440.505 \\ - Fellow de Gastroenterología, Universidad \\ Nacional de Colombia. Bogotá, Colombia \\ 2 Profesor Asociado de Medicina Interna, \\ Universidad Nacional de Colombia, \\ Hospital Universitario Nacional. Unidad de \\ Gastroenterología y Ecoendoscopia (UGEC). \\ Bogotá, Colombia. \\ *Correspondencia: Julián Ernesto Parga-Bermúdez. \\ julianpargab@gmail.com \\ Fecha recibido: $\quad 03 / 02 / 20$ \\ Fecha aceptado: $17 / 06 / 20$

\section{c) $(9)$}

\begin{abstract}
Resumen
La pancreatitis del surco (PS) o "groove pancreatitis", es una forma de pancreatitis crónica infrecuente; nombrada así por su localización en el surco pancreatoduodenal. Suele predominar en varones con antecedente de ingesta de alcohol. Es de gran importancia realizar diagnóstico diferencial con cáncer de páncreas, dada su ubicación. Los avances en los métodos diagnósticos, como la ultrasonografía endoscópica, han permitido un abordaje más certero, sin embargo, no se cuenta con guías de manejo y las aproximaciones terapéuticas siguen siendo basadas en patologías similares.
\end{abstract}

Palabras clave

Pancreatitis, cáncer de páncreas.

\section{Abstract}

Groove pancreatitis (GP) is a rare form of chronic pancreatitis located in the pancreaticoduodenal groove, hence its name. It is predominant in males with a history of alcohol intake. Making a differential diagnosis between this condition and pancreatic cancer is highly relevant given its location. Advances in diagnostic methods, such as endoscopic ultrasonography, have allowed a more accurate approach. However, no management guidelines are available and therapeutic approaches are still based on similar pathologies.

\section{Keywords}

Pancreatitis; Pancreatic cancer.

\section{INTRODUCCIÓN}

La pancreatitis del surco es una forma de pancreatitis crónica infrecuente, nombrada de esta manera por su localización en el surco pancreatoduodenal. Suele predominar en varones con antecedente de ingesta de alcohol. Es de gran importancia realizar un diagnóstico diferencial con cáncer de páncreas, debido a su ubicación. Los avances en los métodos diagnósticos, como la ultrasonografía endoscópica, han permitido un abordaje más certero; sin embargo, no se cuenta con guías de manejo y las aproximaciones terapéuticas siguen siendo basadas en patologías similares.

\section{CASO CLÍNICO}

Se presenta el caso de un varón de 44 años con antecedente de pancreatitis aguda, consumo de alcohol y cigarrillo desde los 20 años, quien consultó a la institución por dolor abdominal localizado en el epigastrio de 6 meses de evolución y se agudizó 4 días antes de su ingreso. Los paraclí- 
nicos evidenciaron elevación de lipasa, fosfatasa alcalina y bilirrubinas con predominio de la directa, lo que configuró un patrón colestásico. La ecografía hepatobiliar mostró el colédoco dilatado de $13 \mathrm{~mm}$, sin evidencia de coledocolitiasis. Ante la situación clínica se realizó colangiorresonancia con engrosamiento difuso concéntrico de las paredes de la 2. ${ }^{a}$ porción del duodeno con masa exofítica irregular dependiente de la pared anterior del mismo, hiperintensa en $\mathrm{T} 1$ e hipointensa en $\mathrm{T} 2$ con restricción en la difusión y una dimensión de 24 × 23 × $24 \mathrm{~mm}$, que condicionaban la obstrucción de la vía biliar intra- y extrahepática.

Se procedió a la realización de ultrasonografía biliopancreática con el equipo Pentax lineal y se encontraron cambios sugestivos de pancreatitis crónica y dilatación del colédoco, pero en la fase endoscópica del examen se observó edema y eritema del bulbo y la segunda porción del duodeno con engrosamiento ecográfico de la pared duodenal que rodea la cabeza del páncreas, dependiente de las ecocapas 1 y 2 , y parcialmente de la 3 ; es decir, de la mucosa y la submucosa; pero estaba conservada la ecoestructura de las capas, sin adenopatías perilesionales. La punción no mostró lesiones que sugirieran neoplasia. Se decidió realizar colangiopancreatografía retrógrada endoscópica (CPRE) y se encontró al paso del duodenoscopio un gran edema, eritema y erosión en la segunda porción del duodeno; y las papilas mayor y menor edematizadas con dilatación del colédoco, pero sin cálculos (Figura 1). Se realizó la colocación de stent biliar para asegurar un adecuado drenaje. Por la sospecha de lesión ampular se tomaron biopsias, las cuales fueron reportadas como "proceso inflamatorio sin evidencia de displasia o neoplasia”. La evolución del paciente fue favorable y se mantiene bajo seguimiento clínico periódico, con tratamiento enzimático oral y recomendaciones nutricionales, por lo cual se consideró que el paciente cursó con una pancreatitis del surco que comprometió principalmente el duodeno y las dos papilas, y generó cambios obstructivos biliares y pancreáticos.

\section{DISCUSIÓN}

El surco pancreático duodenal es una pequeña área confinada entre la cabeza del páncreas, el duodeno y el colédoco (Figura 2) (1). El término pancreatitis de surco (groove pancreatitis) se refiere a un tipo de pancreatitis crónica que afecta principalmente a esta área del páncreas, mientras que el resto del órgano permanece intacto $(2,3)$. Es una entidad poco común, probablemente porque es infradiagnosticada (4).

La entidad fue descrita por primera vez en 1973 por Becker, utilizando el término alemán Rinnenpankreatitis (5). En 1982 Solte y colaboradores lo tradujeron como pancreatitis de surco (2). Posteriormente, una variedad de términos se ha utilizado para referirse a esta entidad en la

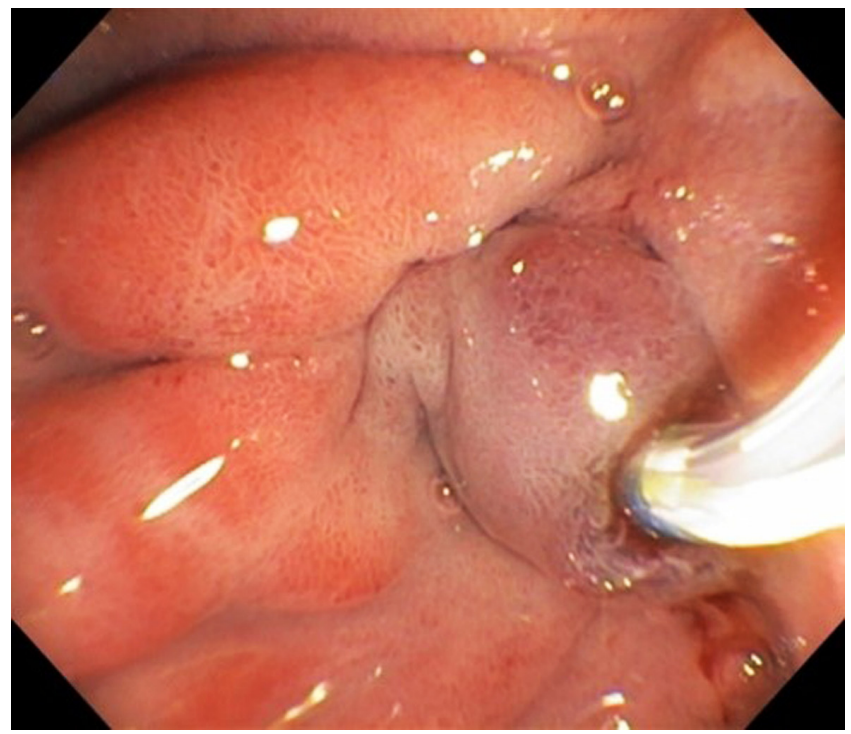

Figura 1. Edema de papilla y región peripapilar.

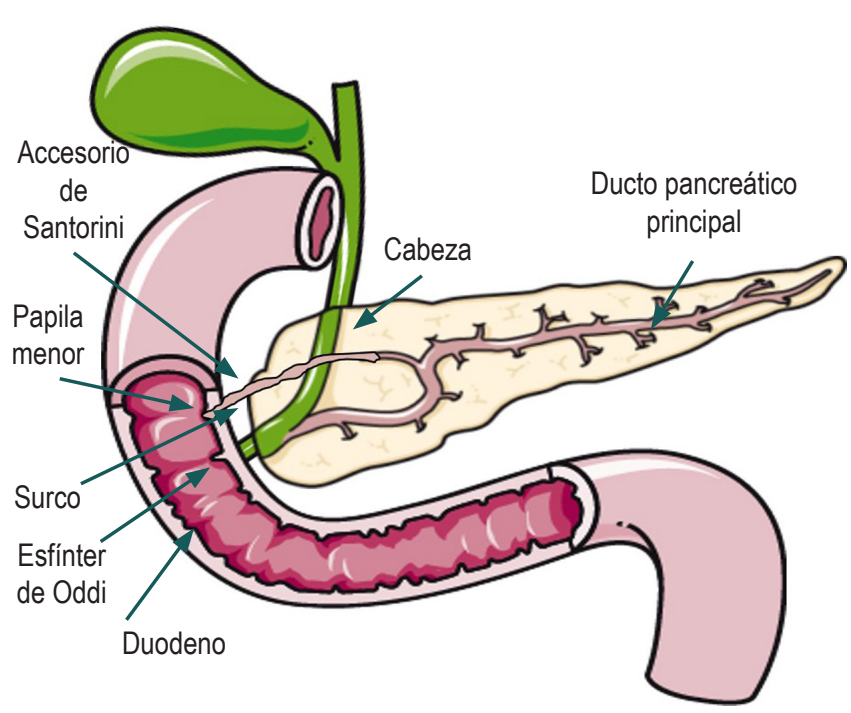

Figura 2. Relaciones anatómicas del surco pancreático.

literatura, como distrofia del páncreas o distrofia duodenal, la cual fue reportada por los autores franceses Potet y Duclert (6). Entre otros, se encuentran distrofia quística heterotópica, quiste de la pared duodenal/paraduodenal, hamartoma pancreático de la pared duodenal, mioadenomatosis, hamartoma de la glándula de Brunner y pancreatitis paraduodenal. Estas diferencias en la terminología hacen difícil su búsqueda en la literatura (7).

En 1991 Becker y Mischke describieron dos formas de pancreatitis del surco: pura y segmentaria (8). En la forma pura, el compromiso infiltrativo o tejido cicatricial afecta solo el surco pancreático, mientras que el parénquima y el 
conducto pancreático principal permanecen preservados. En la forma segmentaria, el tejido cicatricial se extiende a la cabeza y al cuerpo, cerca de la pared duodenal y el conducto pancreático principal, lo que genera estenosis $(9,10)$. La distinción entre las dos formas no siempre es clara (11). La pancreatitis del surco es más común en hombres, con historia de alcoholismo crónico y tiende a aparecer en la cuarta o quinta década de la vida. Algunas series también han asociado el cigarrillo como factor de riesgo $(12,13)$.

La importancia de la pancreatitis del surco está dada por su capacidad de simular al carcinoma de páncreas (10). También hay casos descritos de coexistencia o incluso enmascaramiento de la entidad (14), por lo que debe ser considerada en el diagnóstico diferencial de masas pancreáticas o estenosis duodenal $(15,16)$.

\section{PRESENTACIÓN CLÍNICA}

Clínicamente se presenta con dolor en el epigastrio o mesogastrio, asociado con emesis posprandial, pérdida de peso (principalmente por la alteración de la motilidad intestinal) y estenosis del duodeno $(12,17)$. La presencia de ictericia es usualmente fluctuante, excepto cuando cursa con cáncer de páncreas o colangitis $(18,19)$. El curso de la enfermedad es crónico, y puede ser de años en relación con la aparición de los síntomas y el diagnóstico (20).

La fisiopatología no está claramente definida. Se cree que la entidad está relacionada con la interrupción en el drenaje de jugo pancreático a través del conducto de Santorini, el cual se localiza en el cuerpo del páncreas y drena al conducto de Wirsung formando un ángulo agudo; una vez allí, genera pancreatitis en el área del surco por el aumento de la presión intraductal (15). Además, las alteraciones anatómicas en la papila menor o por disfunción de la papila producen pancreatitis del surco $(3,21)$. Dentro de las primeras están los tumores que ocluyen la papila menor y el conducto de Santorini (7), un conducto de Santorini ocluido, páncreas divisum y páncreas heterotópico en la pared duodenal (15). Las causas funcionales son asociadas con factores precipitantes. En este grupo están la hiperplasia de glándulas de Brunner y el consumo crónico excesivo de alcohol o tabaco, que producen disfunción de la papila menor y aumento en la cantidad de proteínas del jugo pancreático $(3,22)$. En nuestro paciente, el consumo de alcohol y el cigarrillo se ligaron como factores precipitantes de la patología.

La diferenciación macroscópica de la entidad se da en dos escenarios: el tipo quístico, cuyas formaciones de quistes resaltan de la mucosa duodenal más allá de la región ampular, con tamaños que oscilan entre $10 \mathrm{~mm}$ y $10 \mathrm{~cm}$; y el tipo sólido, cuya presentación corresponde a quistes de menos de $10 \mathrm{~mm}$, asociado con un importante engrosamiento de la pared duodenal (23). Adicionalmente, la cicatrización provoca cambios en el colédoco, dejando una superficie lisa, con cambios de hialinización homogénea (7). En estadios tempranos, la pancreatitis del surco afecta levemente la cabeza del páncreas y presenta algunas cicatrices o retracciones. Con la evolución de la enfermedad se produce una importante fibrosis que llega a comprometer la cabeza en su totalidad (23).

Microscópicamente, los hallazgos histológicos incluyen dilatación de los ductos pancreáticos y formación de pseudoquistes en la pared duodenal, fibrosis extensa en la submucosa duodenal con extensión al cuello del páncreas e hiperplasia variable de las glándulas de Brunner, generando una capa gruesa alrededor del músculo liso con proliferación de miofibroblastos (24). Los cambios quísticos se ven en la pared duodenal, atravesando la muscular propia y superficialmente alcanzando el parénquima pancreático. La proliferación miofibroblástica puede encerrar áreas de cambios quísticos con secreciones muy espesas que corresponden a células fusiformes (13). Adicionalmente, los conductos dilatados y el epitelio columnar pueden erosionarse $y$ transformarse en un proceso de fibrosis que da la apariencia de pseudoquiste, lo que configura la variedad quística mencionada $(13,25)$.

\section{DIAGNÓSTICO}

El diagnóstico se construye con un conjunto de pruebas bioquímicas, imágenes y, en algunos casos, toma de biopsias. Los niveles de enzimas pancreáticas (amilasa y lipasa) pueden estar elevados (26). Como la mayoría de las presentaciones son de tipo de inflamatorio y no de características neoplásicas, los marcadores tumorales del tracto gastrointestinal (CA19-9 y antígeno carcinoembrionario) generalmente no presentan elevaciones (10). La ecografía abdominal muestra una lesión hipoecoica que engrosa la pared duodenal y provoca estenosis en la segunda porción del duodeno y obstrucción del colédoco (22). La enfermedad puede ser documentada ecográficamente en varios estadios: en el temprano predomina la fibrosis y se encuentra una banda hipoecoica sobre el surco pancreático que se asocia con engrosamiento de la pared duodenal; mientras que en el tardío, con la fibrosis establecida, hay hiperecogenicidad en la pared duodenal, como resultado de la hipertrofia de la submucosa por hiperplasia de las glándulas de Brunner (27).

\section{IMÁGENES}

La tomografía axial computarizada (TAC) abdominal muestra una lesión laminar hipodensa entre la cabeza del páncreas y el duodeno, cercano a la papila menor, que corresponde a tejido cicatricial $(28,29)$. En la fase de contraste hay un 
retraso en la captación del surco, debido a la alteración en la circulación secundaria al proceso fibrótico (10).

En la forma segmentaria se puede apreciar una lesión hipointensa en la cabeza pancreática, próxima a la pared duodenal. El conducto pancreático principal puede tener discreta dilatación hacia el cuerpo y la cola (30). La vascularización peripancreática suele estar preservada, sin signos de trombosis, incluso en presencia de un compromiso extenso (31). La endoscopia digestiva alta muestra un estrechamiento del lumen en el duodeno, con edema, eritema y mucosa de aspecto polipoide en los estadios más avanzados. Las biopsias de la mucosa duodenal son usualmente inconclusas o muestran un proceso inflamatorio activo sin evidencia de lesiones neoplásicas (24).

La CPRE es un procedimiento técnicamente difícil ya que el posicionamiento del duodenoscopio en presencia del edema del duodeno limita la visualización de la papila. El colédoco distal presenta una estenosis sin compromiso en el conducto pancreático principal. El conducto de Santorini presenta obstrucción por tapones de moco o simplemente por el gran edema perilesional $(4,32)$. En nuestro paciente, los hallazgos endoscópicos mostraron un importante edema del duodeno y la región peripapilar (Figura 3).

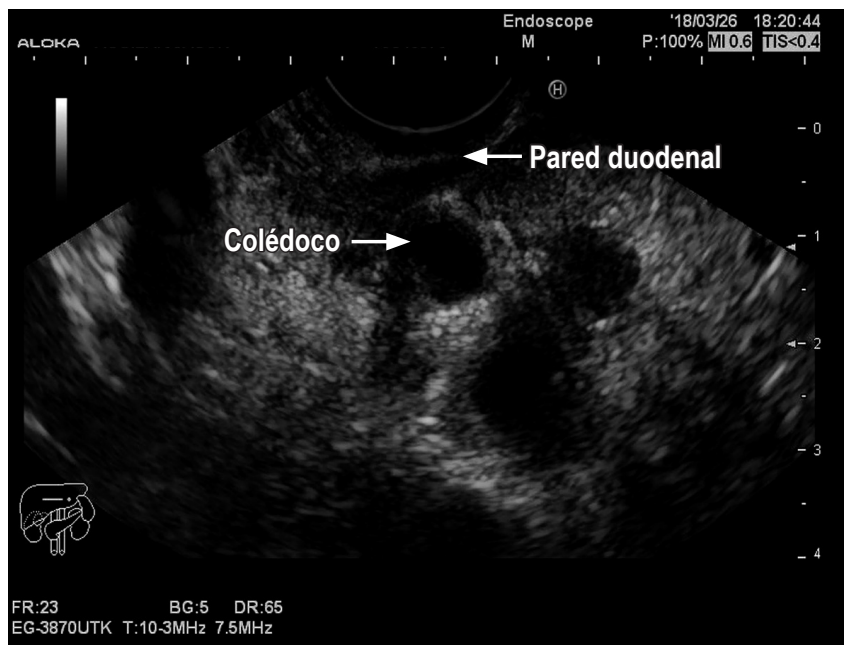

Figura 3. Imagen ecoendoscópica de pared duodenal edematizada.

La ultrasonografía endoscópica (USE) se propone como una técnica diagnóstica de elección debido a una mayor sensibilidad ( $86 \%)$ y con el valor agregado de que posibilita la toma de muestras para el estudio histológico (33). Las imágenes muestran cambios de pancreatitis crónica basada en los criterios de Rosemont, que le brindan un buen rendimiento diagnóstico (34). Al igual que en las otras imágenes, se ha reportado un engrosamiento de la segunda porción del duodeno y heterogeneidad de la parte dorsocraneal de la cabeza del páncreas. Además, en las fases avanzadas hay un hallazgo patognomónico identificado por varios autores: engrosamiento hiperecoico de la pared del duodeno en la región del surco con estructuras ductales anecoicas que se extienden hacia la cabeza. Esta lesión hiperecoica se correlaciona con la proliferación mioadenomatosa y fibrosis del páncreas adyacente $(10,27)$. En nuestro paciente, la ultrasonografía endoscópica evidenció un importante edema de la pared duodenal y dilatación del colédoco (Figura 3).

La resonancia magnética nuclear (RMN) tiene la ventaja de lograr la evaluación de los diferentes aspectos patológicos de la enfermedad. La lesión característica encontrada entre la cabeza del páncreas y el duodeno se observa en la secuencia $\mathrm{T} 1$ como hipointensa, mientras que en $\mathrm{T} 2$ puede adquirir cualquier característica según el tiempo de evolución de la enfermedad (hipo-, iso-, hiperintensa). En los estadios agudos es hiperintensa por el gran edema y en los estadios crónicos es hipointensa por la fibrosis $(28,29)$.

Las lesiones quísticas en el surco o en la pared duodenal, son más evidentes en la secuencia $\mathrm{T} 2$ y es frecuente ver un aumento del grosor o estenosis de la pared duodenal. El colédoco puede presentar estenosis variable. En la forma segmentaria, la cabeza o el páncreas entero pueden verse hipointensos en la secuencia T1, con atrofia del parénquima y dilatación ductal, mostrando la pérdida progresiva de células glandulares que son reemplazadas por tejido fibroso $(29,35)$. La CPRE informa la relación entre el sistema ductal y los cambios quísticos. En la mayoría de los casos se encuentra un ensanchamiento del espacio entre los conductos pancreáticos, colédoco distal y la luz duodenal, por la lesión ocupante de espacio en el surco, así como un marcado engrosamiento de la pared duodenal (28). La colangiorresonancia de nuestro paciente mostró dilatación de la vía biliar, producida por el importante edema en la región peripapilar (Figura 4).

La zona anatómica que ocupa el surco pancreático hace que una lesión localizada en este sitio deba estudiarse con imágenes o histológicamente. El diagnóstico diferencial incluye carcinoma de cabeza de páncreas, carcinoma periampular, tumor neuroendocrino, distrofia quística del duodeno y pancreatitis aguda con plastrón en el surco. La diferenciación de la pancreatitis del surco con el adenocarcinoma es particularmente difícil ya que las dos condiciones pueden tener hallazgos similares $(36,37)$. Una característica importante es la ausencia de compromiso vascular en la pancreatitis del surco. Se ha informado que la arteria gastroduodenal se desplaza hacia la izquierda en la pancreatitis del surco; mientras que, en el carcinoma, se localiza entre la lesión y el duodeno $(38,39)$. Las lesiones quísticas del surco son más comunes en la pancreatitis del surco que en el adenocarcinoma (40). La evaluación duodenal también puede ayudar a diferenciar la pancreatitis del surco del cáncer de páncreas, ya que la 


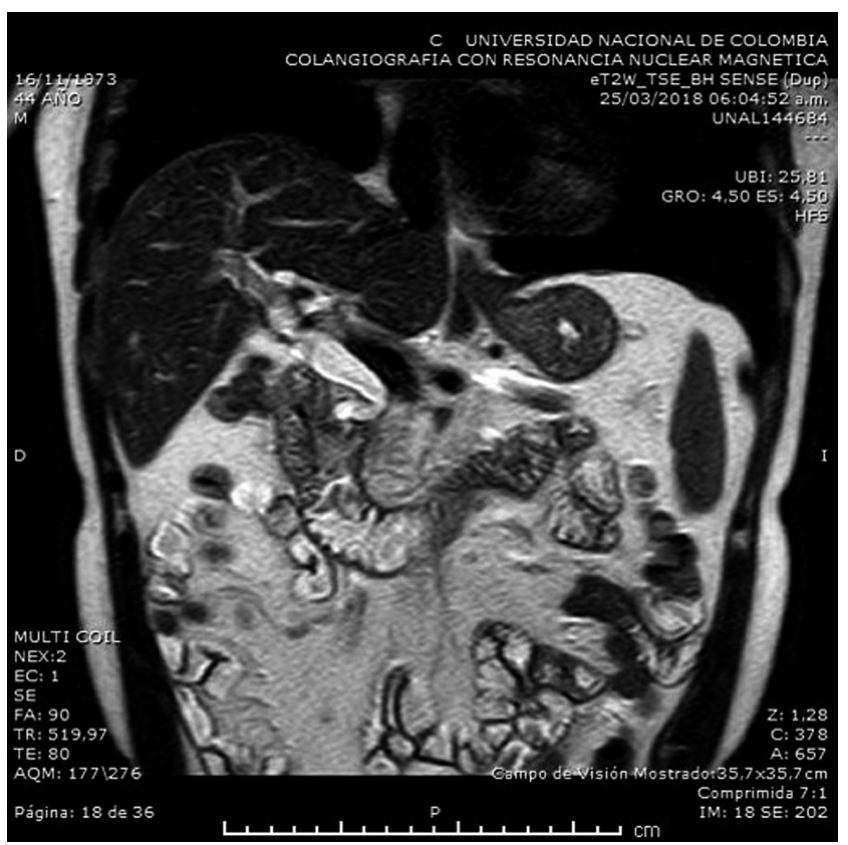

Figura 4. Colangioresonancia con gran edema en segunda porcion duodenal

estenosis es menos común en los tumores de la cabeza del páncreas (35). La colangiorresonancia muestra estenosis distal a la porción intrapancreática del conducto biliar, diferente a la estenosis abrupta e irregular en el adenocarcinoma pancreático (10). Los carcinomas periampulares tienen presentación similar a la del adenocarcinoma ductal de la cabeza del páncreas. Se presentan típicamente en adultos mayores, acompañados de ictericia y pérdida de peso, aunque estos tumores suelen ser adenocarcinomas esclerosantes con alto tejido fibrótico con baja intensidad en las señales de T1 y T2, que causan estenosis del colédoco o terminación abrupta a nivel tumoral que generalmente muestra el signo de hombro, en vez del estrechamiento largo y uniforme visto en la pancreatitis del surco $(41,42)$. El gastrinoma es el tumor neuroendocrino que más comúnmente se localiza en el surco del páncreas. Estos tumores se diferencian de la pancreatitis del surco por su hipervascularidad en imágenes después del contraste, con realce del anillo periférico posterior al uso de gadolinio $(10,43)$.

La distrofia quística de la pared del duodeno se caracteriza por la presencia quistes dentro de la pared duodenal que se origina del tejido pancreático ectópico y sus hallazgos imagenológicos son muy similares a la pancreatitis del surco. Hasta ahora es incierto si la pancreatitis del surco y la distrofia quística del duodeno son entidades distintas o parte del mismo espectro de enfermedad. Por tanto, se conoce en la literatura el término de pancreatitis paraduodenal, en el que se incluye la pancreatitis del surco, la distrofia quística de la pared del duodeno y los quistes paraduodenales de la pared (36).

\section{CONCLUSIÓN}

La pancreatitis del surco es una forma poco común de pancreatitis crónica focal. Las imágenes como la TAC y RMN son modalidades diagnósticas de elección; sin embargo, la llegada de la ecoendoscopia biliopancreática ha permitido tener un acceso a la valoración del páncreas sin radiación y sin exposición a contraste. La intención de este reporte de caso es poner de manifiesto la entidad como diagnóstico diferencial a los episodios agudos de pancreatitis que realmente podrían ser agudizaciones de la una entidad crónica. El conocimiento de las características de la pancreatitis del surco favorece que el médico realice un correcto diagnóstico con menos pruebas diagnósticas invasivas y con potenciales riesgos para el paciente.

\section{REFERENCIAS}

1. Hernandez-Jover D, Pernas JC, Gonzalez-Ceballos S, Lupu I, Monill JM, Pérez C. Pancreatoduodenal junction: review of anatomy and pathologic conditions. J Gastrointest Surg. 2011;15(7):1269-81.

https://doi.org/10.1007/s11605-011-1443-8

2. Stolte M, Weiss W, Volkholz H, Rösch W. A special form of segmental pancreatitis: "groove pancreatitis". Hepatogastroenterology. 1982;29(5):198-208.

3. Tezuka K, Makino T, Hirai I, Kimura W. Groove pancreatitis. Dig Surg. 2010;27(2):149-52. https://doi.org/10.1159/000289099
4. Meesiri S. Groove pancreatitis: report of one case in Thailand. J Med Assoc Thai. 2009;92(11):1554-9.

5. Mohl W, Hero-Gross R, Feifel G, Kramann B, Püschel W, Menges M, Zeitz M. Groove pancreatitis: an important differential diagnosis to malignant stenosis of the duodenum. Dig Dis Sci. 2001 May;46(5):1034-8. https://doi.org/10.1023/a:1010710011767

6. Potet F, Duclert N. Dystrophie kystique sur pancréas aberrant de la paroi duodénale. Arch Fr Mal App Dig. $1970 ; 59(4): 223-38$. 
7. Adsay NV, Zamboni G. Paraduodenal pancreatitis: a clinico-pathologically distinct entity unifying "cystic dystrophy of heterotopic pancreas", "para-duodenal wall cyst", and "groove pancreatitis". Semin Diagn Pathol. 2004;21(4):247-54. https://doi.org/10.1053/j.semdp.2005.07.005

8. Becker V, Mischke U. Groove pancreatitis. Int J Pancreatol. 1991;10(3-4):173-82. https://doi.org/10.1007/BF02924155

9. Kwak SW, Kim S, Lee JW, Lee NK, Kim CW, Yi MS, Kim GH, Kang DH. Evaluation of unusual causes of pancreatitis: role of cross-sectional imaging. Eur J Radiol. 2009;71(2):296-312. https://doi.org/10.1016/j.ejrad.2008.04.006

10. Levenick JM, Gordon SR, Sutton JE, Suriawinata A, Gardner TB. A comprehensive, case-based review of groove pancreatitis. Pancreas. 2009;38(6):e169-75. https://doi.org/10.1097/MPA.0b013e3181ac73f1

11. Raman SP, Salaria SN, Hruban RH, Fishman EK. Groove pancreatitis: spectrum of imaging findings and radiology-pathology correlation. AJR Am J Roentgenol. 2013;201(1):W29-39. https://doi.org/10.2214/AJR.12.9956

12. Rahman SH, Verbeke CS, Gomez D, McMahon MJ, Menon KV. Pancreatico-duodenectomy for complicated groove pancreatitis. HPB (Oxford). 2007;9(3):229-34. https://doi.org/10.1080/13651820701216430

13. Casetti L, Bassi C, Salvia R, Butturini G, Graziani R, Falconi M, Frulloni L, Crippa S, Zamboni G, Pederzoli P. "Paraduodenal" pancreatitis: results of surgery on 58 consecutives patients from a single institution. World J Surg. 2009;33(12):2664-9. https://doi.org/10.1007/s00268-009-0238-5

14. Malde DJ, Oliveira-Cunha M, Smith AM. Pancreatic carcinoma masquerading as groove pancreatitis: case report and review of literature. JOP. 2011;12(6):598-602.

15. Shudo R, Yazaki Y, Sakurai S, Uenishi H, Yamada H, Sugawara K, Okamura M, Yamaguchi K, Terayama H, Yamamoto Y. Groove pancreatitis: report of a case and review of the clinical and radiologic features of groove pancreatitis reported in Japan. Intern Med. 2002;41(7):537-42. https://doi.org/10.2169/internalmedicine.41.537

16. Viñolo Ubiña C, Morales Ruiz J, Heredia Carrasco C, RuizCabello Jiménez M, Villegas Herrera MT, Garrote Lara D. Groove pancreatitis with duodenal stenosis. Rev Esp Enferm Dig. 2010;102(1):59-60. https://doi.org/10.4321/S1130-01082010000100012

17. Balakrishnan V, Chatni S, Radhakrishnan L, Narayanan VA, Nair P. Groove pancreatitis: a case report and review of literature. JOP. 2007;8(5):592-7.

18. Rebours V, Lévy P, Vullierme MP, Couvelard A, O’Toole D, Aubert A, Palazzo L, Sauvanet A, Hammel P, Maire F, Ponsot P, Ruszniewski P. Clinical and morphological features of duodenal cystic dystrophy in heterotopic pancreas. Am J Gastroenterol. 2007;102(4):871-9. https://doi.org/10.1111/j.1572-0241.2007.01091.x
19. Kim JD, Han YS, Choi DL. Characteristic clinical and pathologic features for preoperative diagnosed groove pancreatitis. J Korean Surg Soc. 2011;80(5):342-7. https://doi.org/10.4174/jkss.2011.80.5.342

20. German V, Ekmektzoglou KA, Kyriakos N, Patouras P, Kikilas A. Pancreatitis of the gastroduodenal groove: a case report. Case Rep Med. 2010;2010:329587. https://doi.org/10.1155/2010/329587.

21. Isayama H, Kawabe T, Komatsu Y, Sasahira N, Toda N, Tada M, Nakai Y, Yamamoto N, Hirano K, Tsujino T, Yoshida $\mathrm{H}$, Omata M. Successful treatment for groove pancreatitis by endoscopic drainage via the minor papilla. Gastrointest Endosc. 2005;61(1):175-8. https://doi.org/10.1016/s0016-5107(04)02460-5

22. Manzelli A, Petrou A, Lazzaro A, Brennan N, Soonawalla $Z$, Friend P. Groove pancreatitis. A mini-series report and review of the literature. JOP. 2011;12(3):230-3.

23. Zamboni G, Capelli P, Scarpa A, Bogina G, Pesci A, Brunello E, Klöppel G. Nonneoplastic mimickers of pancreatic neoplasms. Arch Pathol Lab Med. 2009; 133(3):439-53. https://doi.org/10.1043/1543-2165-133.3.439

24. DeSouza K, Nodit L. Groove pancreatitis: a brief review of a diagnostic challenge. Arch Pathol Lab Med. 2015;139(3):417-21. https://doi.org/10.5858/arpa.2013-0597-RS

25. Klöppel G. Chronic pancreatitis, pseudotumors and other tumor-like lesions. Mod Pathol. 2007 Feb;20 Suppl 1:S113-31. https://doi.org/10.1038/modpathol.3800690

26. Chute DJ, Stelow EB. Fine-needle aspiration features of paraduodenal pancreatitis (groove pancreatitis): a report of three cases. Diagn Cytopathol. 2012;40(12):1116-21. https://doi.org/10.1002/dc.21722

27. Wronski M, Karkocha D, Slodkowski M, Cebulski W, Krasnodebski IW. Sonographic findings in groove pancreatitis. J Ultrasound Med. 2011;30(1):111-5. https://doi.org/10.7863/jum.2011.30.1.111

28. Arora A, Dev A, Mukund A, Patidar Y, Bhatia V, Sarin SK. Paraduodenal pancreatitis. Clin Radiol. 2014;69(3):299-306. https://doi.org/10.1016/j.crad.2013.07.011

29. Perez-Johnston R, Sainani NI, Sahani DV. Imaging of chronic pancreatitis (including groove and autoimmune pancreatitis). Radiol Clin North Am. 2012;50(3):447-66. https://doi.org/10.1016/j.rcl.2012.03.005

30. Zaheer A, Haider M, Kawamoto S, Hruban RH, Fishman EK. Dual-phase CT findings of groove pancreatitis. Eur J Radiol. 2014;83(8):1337-43. https://doi.org/10.1016/j.ejrad.2014.05.019

31. Coakley FV, Hanley-Knutson K, Mongan J, Barajas R, Bucknor M, Qayyum A. Pancreatic imaging mimics: part 1, imaging mimics of pancreatic adenocarcinoma. AJR Am J Roentgenol. 2012;199(2):301-8. https://doi.org/10.2214/AJR.11.7907

32. Sanada Y, Yoshida K, Itoh H, Kunita S, Jinushi K, Matsuura $\mathrm{H}$. Groove pancreatitis associated with true pancreatic cyst. 
J Hepatobiliary Pancreat Surg. 2007;14(4):401-9. https://doi.org/10.1007/s00534-006-1180-7

33. Dominguez-Muñoz JE. Pancreatitis aguda. Gastroenterol Hepatol. 2006;29(3):77-84. https://doi.org/10.1157/13098300

34. Dimcevski G, Erchinger FG, Havre R, Gilja OH. Ultrasonography in diagnosing chronic pancreatitis: new aspects. World J Gastroenterol. 2013;19(42):7247-57. https://doi.org/10.3748/wjg.v19.i42.7247

35. Blasbalg R, Baroni RH, Costa DN, Machado MC. MRI features of groove pancreatitis. AJR Am J Roentgenol. 2007;189(1):73-80. https://doi.org/10.2214/AJR.06.1244

36. Shanbhogue AK, Fasih N, Surabhi VR, Doherty GP, Shanbhogue DK, Sethi SK. A clinical and radiologic review of uncommon types and causes of pancreatitis. Radiographics. 2009;29(4):1003-26. https://doi.org/10.1148/rg.294085748

37. Chatelain D, Vibert E, Yzet T, Geslin G, Bartoli E, Manaouil D, Delcenserie R, Brevet M, Dupas JL, Regimbeau JM. Groove pancreatitis and pancreatic heterotopia in the minor duodenal papilla. Pancreas. 2005;30(4):e92-5. https://doi.org/10.1097/01.mpa.0000161885.79373.1d

38. Graziani R, Tapparelli M, Malagò R, Girardi V, Frulloni L, Cavallini G, Pozzi Mucelli R. The various imaging aspects of chronic pancreatitis. JOP. 2005 Jan 13;6(1 Suppl):73-88.
39. Triantopoulou C, Dervenis C, Giannakou N, Papailiou J, Prassopoulos P. Groove pancreatitis: a diagnostic challenge. Eur Radiol. 2009;19(7):1736-43. https://doi.org/10.1007/s00330-009-1332-7

40. Castell-Monsalve FJ, Sousa-Martin JM, Carranza-Carranza A. Groove pancreatitis: MRI and pathologic findings. Abdom Imaging. 2008;33(3):342-8. https: / / doi.org/10.1007/s00261-007-9245-x

41. Irie $H$, Honda $H$, Kuroiwa $T$, Hanada $K$, Yoshimitsu $K$, Tajima T, Jimi M, Yamaguchi K, Masuda K. MRI of groove pancreatitis. J Comput Assist Tomogr. 1998;22(4):651-5. https://doi.org/10.1097/00004728-199807000-00027

42. Fulcher AS, Turner MA. HASTE MR cholangiography in the evaluation of hilar cholangiocarcinoma. AJR Am J Roentgenol. 1997;169(6):1501-5. https://doi.org/10.2214/ajr.169.6.9393153

43. Semelka RC, Custodio CM, Cem Balci N, Woosley JT. Neuroendocrine tumors of the pancreas: spectrum of appearances on MRI. J Magn Reson Imaging. 2000; 11(2):141-8. https://doi.org/10.1002/(SICI)15222586(200002)11:2<141::AID-JMRI10>3.0.CO;2-U 